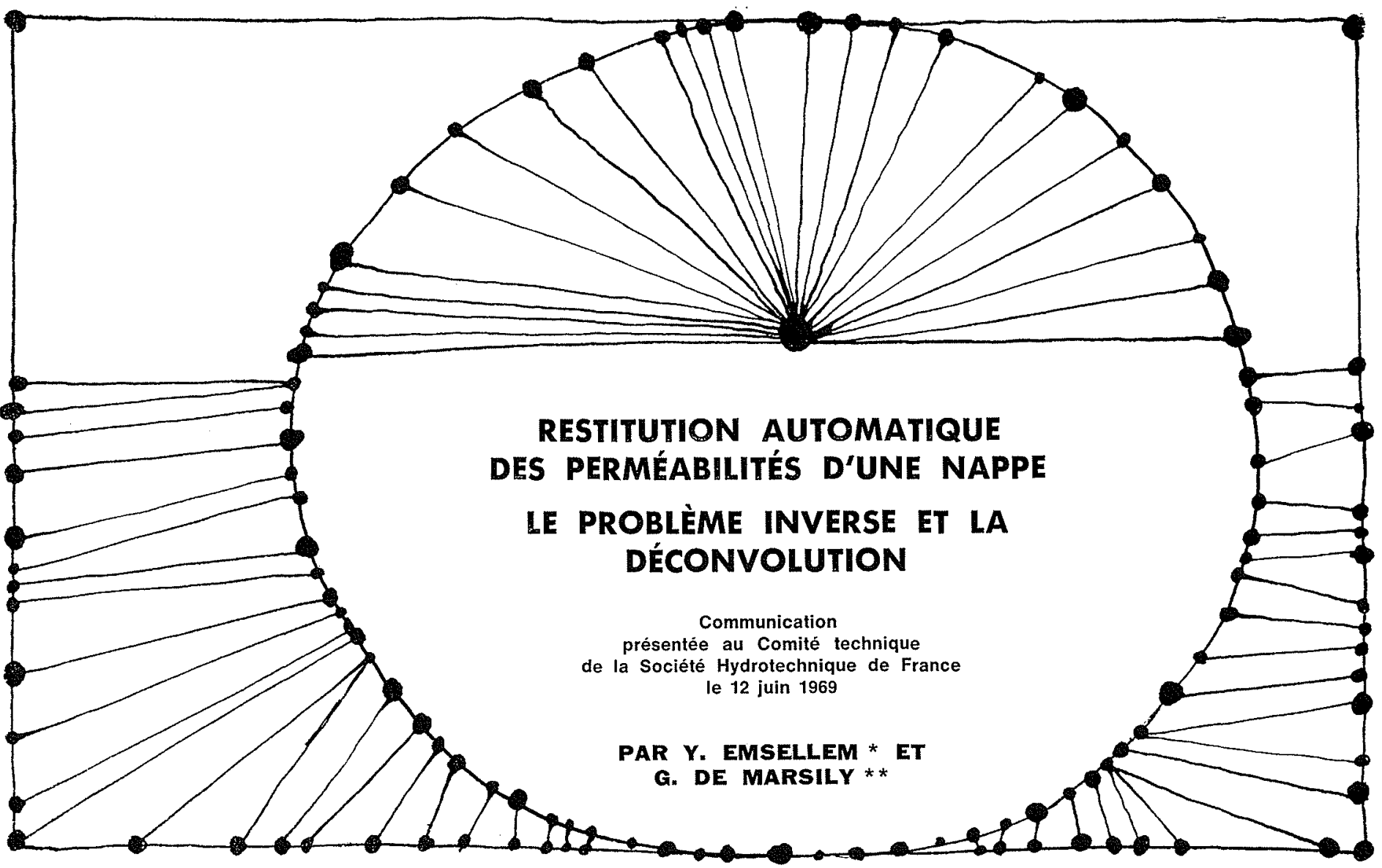

Le problème de l'interprétation des informations se pose souvent dans les termes suivants :

Un système physique, dont les caractéristiques sont inconnues, a pour effet de transformer un phénomène d'entrée $s$ en une grandeur de sortie $\mathrm{S}$.

Par exemple, le pompage dans divers puits de débits constants amène, à l'équilibre, la piézométrie de la nappe exploitée à un niveau $\mathrm{H}(x, y, z)$.

De mème, un traceur pénètre dans un tube poreux avec une concentration à l'entrée $c(t)$ : il sort, du fait de la dispersion par exemple, un liquide de concentration $\mathrm{C}(t)$.

La question que l'on se pose est :

- dans le premier cas, quelle est la distribution de la transmissivité $T$, satisfaisant à :

$$
\operatorname{div} \mathrm{T} \operatorname{grad} \mathrm{H}=\mathrm{Q}
$$

- dans le second : quelle est la fonction «opérateur » $p(t)$ qui est la réponse à l'injection instantanée d'une masse de traceur unitaire $U$, telle que par conséquent:

$$
\mathrm{C}(\boldsymbol{t})=\int_{0}^{t} p(x) c(t-x) d x
$$

Les deux problèmes ont la même structure car l'on cherche des informations sur l'opérateur, c'est-à-dire le système physique, représenté soit par une description de sa consistance (T), soit par sa réponse à une sollicitation élémentaire (p).

La méthode que nous allons exposer part de la constatation que les techniques itératives de calcul

\footnotetext{
* Arrondissement minéralogique de Bordeaux.
}

* E Ecole des Nines, Paris. utilisées (analyse de Fourier notamment) pour obtenir une représentation ponctuelle, c'est-à-dire une transmissivité par point ou une valeur par époque de la réponse unitaire, dès la première itération ont une tendance à l'instabilité. Cette instabilité est caractérisée par le fait que deux valeurs voisines du résultat ont fréquemment un signe différent ce qui est absurde physiquement, et que la moyenne locale des valeurs de plusieurs points voisins est très inférieure à l'amplitude des variations locales.

La technique de calcul mise au point procède par itération en modifiant les valeurs de la grandeur inconnue par plages de dimensions décroissantes.

De plus, toute modification ponctuelle ne peut découler que d'une modification d'ensemble portant sur la totalité du domaine support de la grandenr inconnue.

Nous allons exposer le principe de la méthode, puis en présenter trois applications, l'une à la déconvolution, l'autre à la restitution automatique des perméabilités (problème inverse), l'autre au problème direct.

Le problème direct consiste à calculer la pression $H$ connaissant la transmissivité $T$, le problème inverse la transmissivité, connaissant la pression, $\mathrm{T}$ et $\mathrm{H}$ étant liés par :

$$
\operatorname{div} \mathrm{T} \operatorname{grad} \mathrm{H}=\mathrm{Q}
$$

Principe

Appelons $p$ un opérateur qui fait passer une entrée $s$ à une sortie $\mathrm{S}$, ce que nous noterons conventionnellement :

$$
\mathrm{S}=p * s
$$




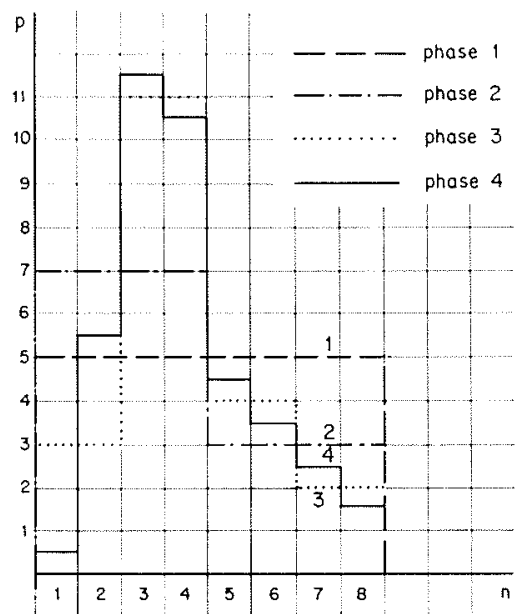

1/ Déconvolution. Principe de la méthode.

Principle of deconvolution method.

2/ Eléments de base / Busic functions.

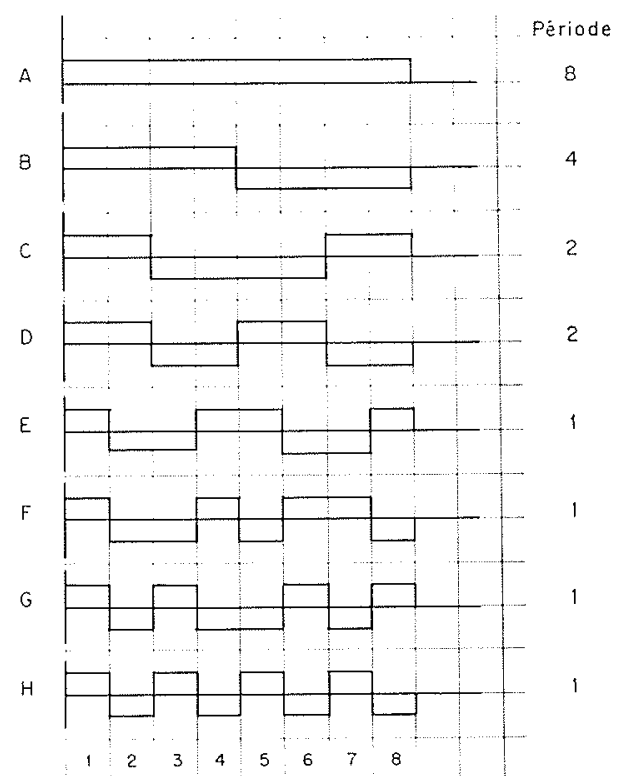

On peut alors écrire :

$p=a \mathrm{~A}+b \mathrm{~B}+c \mathrm{C}+d \mathrm{D}+e \mathrm{E}+f \mathrm{~F}+g \mathrm{G}+h \mathrm{H}$

Pour l'exemple choisi :

$$
\begin{aligned}
& a=5[5+2=7 \text { (moyenne }(1-4))] \\
& b=2[5-2=3(\text { moyenne }(5-8))]
\end{aligned}
$$
elc.

D'autre part, à A, B,.. G, H, il correspond :

$$
\mathrm{A} * s, \ldots, \mathrm{G} * s
$$

Définissons d'autre part les vecteurs $\mathrm{S}$ et $s$. Les observations sur $S$ et $s$ sont discrètes; on peut numéroter leurs occurrences et représenter $S$ et $s$ par deux vecteurs :

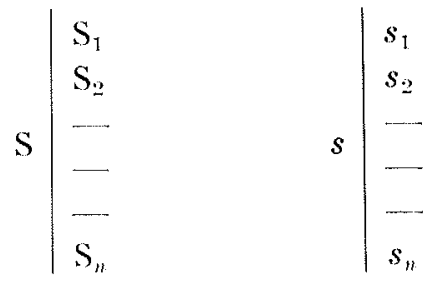

alors la norme de $\mathrm{S}$ et de $s$ est définie par :

$$
\begin{aligned}
& \|\mathrm{S}\|^{2}=\mathrm{S}^{2}=\sum_{1}^{n}\left(\mathrm{~S}_{i}\right)^{2} \\
& \mathrm{~s}^{2}=s^{2}=\sum_{1}^{n}\left(s_{i}\right)^{2}
\end{aligned}
$$

Le produit scalaire de $\mathrm{S}$ et $\mathrm{R}$ est, de mème :

$$
\mathrm{S} \mathrm{R}=\sum_{i}^{l i} \mathrm{~S}_{i} \mathrm{R}_{i}
$$

Nous allons chercher à présent $p$.

Nous commencons par rechercher une approximation $a_{1}$ de $a$, en minimisant la somme de carrés :

$$
\left(\mathrm{S}-a_{1} \mathrm{~A} * s\right)^{2}
$$


done :

$$
a_{1}=\frac{(\mathrm{A} * s) \mathrm{S}}{(\mathrm{A} * s)^{2}}
$$

$a_{1}$ étant connu à présent, nous cherchons une approximation $b_{1}$ de $b$ en minimisant la somme de carrés :

done :

$$
b_{1}^{\text {min }}=\left(\mathrm{S}--a_{1} \mathrm{~A} * s-b_{1} \mathrm{~B} * s\right)
$$

$$
b_{1}=\frac{(\mathrm{B} * s)\left(\mathrm{S}-a_{1} \mathrm{~A} * s\right)}{\left(\mathrm{B} * s^{2}\right)}
$$

et ainsi de suite.

Une fois épuisé l'ensemble $A, B, C, \ldots H$, on recommence à partir de $\mathrm{A}$.

La méthode consiste donc à construire une hiérarchie de $\mathrm{A}$ à $\mathrm{H}$ en décidant que l'on cherchera d'abord à expliquer le maximum de $\mathrm{S}$ et $s$ à partir d'un élément constant sur tout le domaine A, puís un élément plus variable $B$ et ainsi de suite. De la sorte, la contribution des éléments oscillants tels que $\mathbf{H}$ ne sera évaluée que lorsque le maximum aura été fait pour obtenir une explication à partir d'éléments moins oscillants.

\section{Le noyau.}

Prenons un opérateur $q$ à $m$ composantes pour une entrée $s$ à $m$ composantes; il peut se faire que lorsque l'on n'examine $\mathrm{S}$ (c'est-à-dire $q * s$ ) que sur' $m$ composantes :

$$
q * s=0
$$

$q$ fait alors partie du noyau de déconvolution attaché à $s$, de même que $s$ fait partie du noyau de convolution attaché à $q$. Mais en général, en effectue la recherche de $q$ en utilisant un grand nombre $n$ de données très supérieures à $m, s$ et $S$ ayant beaucoup plus de $m$ composantes : il est alors exclu d'obtenir un $q$ tel que, pour les $n$ composantes de $\mathrm{S}$ :

$$
\mathrm{S}=q * s=0
$$

sur la totalité, l'intervalle de longueur $n$.

Ceci signifie que les $n$ équations à $m$ inconnues $a, \quad \mathrm{~b}, \ldots$ correspondant à $\mathrm{S}=(a \mathrm{~A}+b \mathrm{~B}+\ldots) * s$ doivent être compatibles et n'admettent alors qu'une seule solution. Pour cela, il faut que $\mathrm{A} * s$, $B * s$, etc., soient linéairement indépendantes et que $S$ soit leur combinaison avec les poids respectifs $a$, $b, \ldots$ etc.

Le problème du noyau, c'est-à-dire de l'indétermination, ne se pose done que lorsque le nombre de coordonnées de l'entrée et de la sortie est inférieur ou égal au nombre de composantes de l'opérateur. Nous verrons avec le problème inverse la signification de la solution obtenue lorsque le noyau ne se réduit pas à $q=0$.

\section{Unicité de la solution.}

On sait, à présent, que dans les cas pratiques $\mathrm{A} * s, \mathrm{~B} * s$, etc., sont indépendants et que les composantes de S sur ces recteurs sont $a, b, \ldots$

Nous allons vérifier que l'opérateur $\mathrm{P}_{n}$ :

$$
\mathrm{P}_{n}=a_{n} \mathrm{~A}+b_{\mathrm{N}} \mathrm{B}+\cdots
$$

tend vers $p$ quand $n$ tend vers l'infini.
Tout d'abord, la norme de $\left(\mathrm{S}-\mathrm{P}_{n} * s\right)$ ne peut que décroitre. Elle a donc une limite, qui est positive ou nulle. Appelons $\mathrm{T}_{i}$ un élément de base, c'est-à-dire :

$$
\begin{aligned}
& \mathrm{T}_{1}=\mathrm{A} \\
& \mathrm{T}_{2}=\mathrm{B}
\end{aligned}
$$

etc.

Appelons enfin $d_{n}$ l'erreur commise au pas $n$ :

$$
d_{n}=\mathrm{S}-\mathrm{P}_{n} * s
$$

la norme $\left\|d_{n}\right\|=\left(\mathrm{S}-\mathrm{P}_{n} * s\right)^{2}$ tend vers une limite $\|d\|$. Montrons qu'elle est nulle.

En effet :

$$
\begin{gathered}
d_{n+1}=\mathrm{S}-\mathrm{P}_{n+1} * s=\left(\mathrm{S}-\mathrm{P}_{n} * s\right) \\
-\frac{\left(\mathrm{S}-\mathrm{P}_{n} * s\right) \mathrm{T}_{i} * s}{\left(\mathrm{~T}_{i} * s\right)^{2}} \mathrm{~T}_{i} * s \\
d_{n+1}=d_{n} \frac{d_{n} \mathrm{~T}_{i} * s}{\left(\mathrm{~T}_{i} * s\right)^{2}}\left(\mathrm{~T}_{i} * s\right)
\end{gathered}
$$

donc :

$$
\left\|d_{n+1}\right\|=\left\|d_{n}\right\|-\frac{\left(d_{n}-\mathrm{T}_{i} * s\right)^{2}}{\left(\mathrm{~T}_{i} * s\right)^{2}}
$$

si :

alors :

$$
\begin{aligned}
n & \rightarrow \infty \\
\left\|d_{n}\right\| & \rightarrow\|d\| \\
\left\|d_{n+1}\right\| & \rightarrow\|d\|
\end{aligned}
$$

$$
d_{n} \mathbf{T}_{i} * s=0
$$

$d_{n}$ est donc orthogonal à $\mathrm{T}_{i} * s$ quel que soit $\mathrm{T}_{i}$ et comme $d_{n}$ n'a de composante que sur les $\mathbf{T}_{i} * s, d_{n}$ est nul.

On aboutit donc bien au résultat cherché.

En résumé, la solution est unique dès que le nombre de coordonnées de l'entrée et de la sortie est supérieur à celui de l'opérateur. Dans la pratique, les mesures de $\mathrm{S}$ et de $s$ sont entachées d'erreur. Le système :

$$
\mathrm{S}=p * s
$$

n'a donc plus une solution unique, mais le fait de disposer d'un grand nombre de données sur $S$ et $s$ fait que la recherche du minimum de:

$$
(\mathrm{S}-p * s)^{2}
$$

fournit, en minimisant globalement l'erreur d'interprétation, la solution la plus probable en $p$. Cette optique est très différente d'une simple méthode de moindres carrés car l'ajustement ne se fait qu'après qu'un schéma déterministe ait été construit. Le coefficient de corrélation sortie calculée/ sortie réelle, est supérieur au coefficient de corrélation entrée/sortie.

\section{Déconvolution}

$s(t)$ étant l'entrée, l'opérateur $p(t)$, la sortie $\mathrm{S}(t)$ est égale à :

$$
\mathrm{S}(t)=\int_{1}^{t} p(x) s(t-x) d x
$$




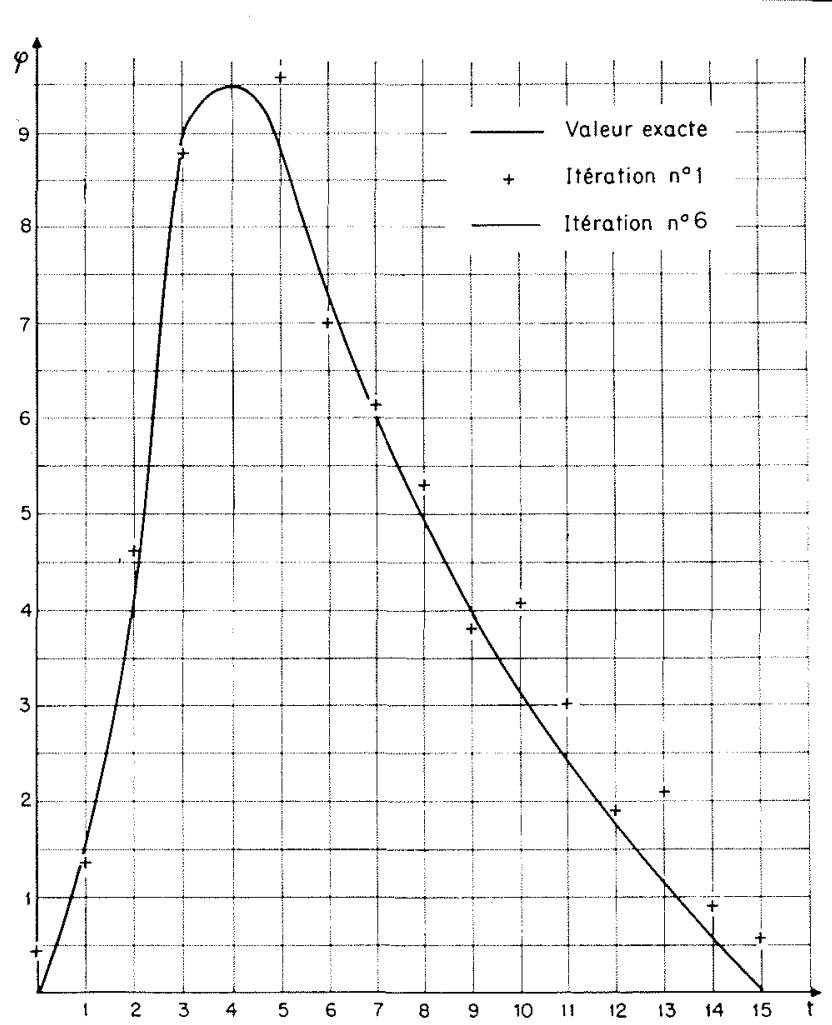

3/ Résultat de la déconvolution. Exemple.

Example of a result.

Supposons que $p(t)$ soit nul en dehors d'un ensemble d'intervalles de longueur totale finie. Prenons pour $S$ un intervalle de variation $(a, b)$ incluant tous ces intervalles. Le problème : recherche de $p$ connaissant $\mathrm{S}$ et $s$, est équivalent à minimiser, pour le meilleur $p$ :

$$
\int_{a}^{b}\left[\mathrm{~S}(t)-\int_{0}^{t} p s(t-x) d x\right]^{2} d t
$$

on construit donc la base $\mathrm{A}, \mathrm{B}$, etc. :

pour : $a \leqslant t \leqslant b$

$$
A=1
$$$$
\mathrm{B}=1
$$

pour : $a \leqslant t<a+\frac{b-a}{2}$

$$
\mathrm{B}=\mathrm{B}-1
$$

pour : $a+\frac{b-a}{2}<t \leqslant b$

etc.

\section{Application.}

Supposons les données $s_{1}, s_{2} \ldots s_{n}$ et $\mathrm{S}_{1}, \ldots \mathrm{S}_{p}$ discrètes, alors :

$$
\mathrm{S}_{i}=\sum_{k=-1}^{i} p_{k} s-k
$$

la base $\mathrm{A}, \mathrm{B}$ sert à construire $\mathrm{A} * s$, etc., et l'on opère comme précédemment.

\begin{tabular}{|c|c|c|c|}
\hline$N^{0}$ & $\underset{\text { exact }}{p}$ & $\mathrm{~N}^{\circ}$ & $\stackrel{s}{\text { exact }}$ \\
\hline 1 & 0 & 1 & 1 \\
\hline 2 & 1,5 & 2 & 2 \\
\hline 3 & 4 & 3 & 4 \\
\hline 4 & 9 & 4 & 5 \\
\hline 5 & 9,55 & 5 & 3 \\
\hline 6 & 8,8 & 6 & 7 \\
\hline 7 & 7,3 & 7 & 0 \\
\hline 8 & 6,1 & 8 & 0 \\
\hline 9 & 5 & 9 & 0 \\
\hline 10 & 4 & 10 & 6 \\
\hline 11 & 3 & 11 & 4 \\
\hline 12 & 2,4 & 12 & 2 \\
\hline 13 & 1,8 & 13 & 3 \\
\hline 14 & 1,2 & 14 & 1 \\
\hline 15 & 0,6 & 15 & 0 \\
\hline 16 & 0 & 16 & 0 \\
\hline
\end{tabular}

\section{Exemple :}

\begin{tabular}{|c|c|c|c|c|c|}
\hline $\mathrm{N}^{\circ}$ & S exact & $\mathrm{N}^{\circ}$ & $S$ exact & $\mathrm{N}^{\circ}$ & $S$ exact \\
\hline 1 & 0 & 11 & 154,2 & 21 & 56,3 \\
\hline 2 & 1,5 & 12 & 199,2 & 22 & 43,8 \\
\hline 3 & 7 & 13 & 170,1 & 23 & 32,8 \\
\hline 4 & 23 & 14 & 182,9 & 24 & 22,4 \\
\hline 5 & 51 & 15 & 182,1 & 25 & 12,6 \\
\hline 6 & 88,3 & 16 & 173,6 & 26 & 6,6 \\
\hline 7 & 130,4 & 17 & 151,5 & 27 & 3,0 \\
\hline 8 & 158,4 & 18 & 124,1 & 28 & 0,6 \\
\hline 9 & 181,9 & 19 & 97,1 & 29 & 0 \\
\hline 10 & 167,8 & 20 & 75 & 30 & \\
\hline
\end{tabular}

Nous avons convolué l'entrée $s$ théorique avec. l'opérateur $p$ pour obtenir $F$.
La composition est :

\begin{tabular}{|c|c|c|c|c|c|c|c|}
\hline$n$ & $\mathrm{P}_{1}$ & $\mathrm{P}_{2}$ & $P_{3}$ & $P_{4}$ & $P_{5}$ & $P_{B}$ & $\begin{array}{c}p \\
\text { réel }\end{array}$ \\
\hline \multicolumn{4}{|c|}{$1|0,453|-0,064 \mid-0,038$} & \multicolumn{4}{|c|}{$-0,015|-0,006|-0,003 \mid 0$} \\
\hline $2 \mid$ & $\mid 1,393$ & 1,328 & 1,407 & 1,451 & 1,474 & \multicolumn{2}{|c|}{$1,486 \mid 1,5$} \\
\hline $3 \mid$ & 4,599 & 4,216 & $\overline{4,114}$ & 4,061 & 4,033 & \multicolumn{2}{|c|}{$4,017 \mid 4$} \\
\hline 4 & 8,686 & 8,826 & $\overline{8,927}$ & 8,967 & 8,984 & \multicolumn{2}{|c|}{$8,993 \mid 9$} \\
\hline & 10,078 & $9,574 \mid$ & 9,504 & 9,497 & 9,497 & \multicolumn{2}{|c|}{$9,498 \mid 9,5$} \\
\hline 6 & 9,751 & 9,114 & 8,945 & 8,875 & 8,840 & \multicolumn{2}{|c|}{$8,821 \mid 8,8$} \\
\hline 7 & 6,972 & 7,054 & 7,147 & 7,218 & 7,257 & \multicolumn{2}{|c|}{$7,278 \mid 7,3$} \\
\hline $8 \mid$ & 6,129 & 6,184 & 6,125 & 6,107 & 6,101 & \multicolumn{2}{|c|}{$6,099 \mid 6,1$} \\
\hline $9 \mid$ & 5,338 & $5,155 \mid$ & 5,072 & 5,039 & 5,022 & \multicolumn{2}{|c|}{$5,013 \mid 5$} \\
\hline 10 & 3,769 & 3,755 & $\overline{3,849}$ & 3,920 & 3,959 & \multicolumn{2}{|c|}{$3,979 \mid 4$} \\
\hline 11 & 4,013 & 3,480 & 3,329 & 3,266 & 3,234 & \multicolumn{2}{|c|}{$3,217 \mid 3,2$} \\
\hline 121 & 2,987 & 2,532 & 2,443 & 2,422 & 2,413 & \multicolumn{2}{|c|}{$2,408 \mid 2,4$} \\
\hline 13 & 1,449 & 1,576 & 1,701 & 1,752 & 1,775 & \multicolumn{2}{|c|}{$1,797 \mid 1,8$} \\
\hline $14 \mid$ & 1,617 & 1,381 & 1,305 & 1,258 & 1,230 & \multicolumn{2}{|c|}{$1,216 \mid 1,2$} \\
\hline 15 & 0,914 & 0,490 & 0,533 & 0,568 & 0,585 & \multicolumn{2}{|c|}{$0,593 \mid 0,6$} \\
\hline$|16|$ & 0,632 & 0,072 & 0,057 & $-0,027$ & $-0,014$ & \multicolumn{2}{|c|}{$-0,008 \mid 0$} \\
\hline
\end{tabular}

$$
\mathrm{S}_{i}=\sum_{k=1}^{i} p_{k} \mathrm{~S}_{i-k+1}
$$

Les résultats ont été les suivants pour les différentes itérations : 
Soulignons de plus que nous n'avons pas intentionnellement utilisé de méthode d'accélération de la convergence, par exemple Aitken ou surrelaxation.

\section{Le problème inverse}

La structure du problème a fait l'objet d'un précédent article ( ${ }^{*}$ ) nous n'y reviendrons pas et nous nous contenterons d'en résumer l'essentiel.

L'équation de base :

$$
\operatorname{div} \mathrm{T} \operatorname{grad} \mathrm{H}=\mathrm{Q}
$$

devient, après discrétisation sur un système à $i$ mailles intérieures et mailles frontières à potentiel constant :

$$
(\mathrm{T}) * \overline{\mathrm{H}}=\overline{\mathrm{Q}}
$$

lorsque l'on appelle :

$\overline{\mathrm{H}}$ le vecteur pression (i coordonnées);

$\bar{Q}$ le vecteur débit ( $i$ coordonnées).

(*) Présentation d'une méthode nouvelle de résolution du problème inverse. Y. Emsellem et G. de Marsily, Jounées H. Scholler, Congres de Bordeaux, avril 1969.
Appelons T le vecteur transmissivité possédant $(i+f)$ mailles; à ce vecteur $\overline{\mathrm{T}}$ correspond un opérateur (T) qui fait passer de $\mathrm{H}$ à $\mathrm{Q}$ en équilibre le bilan des flux transitant à travers chacune des $i$ mailles.

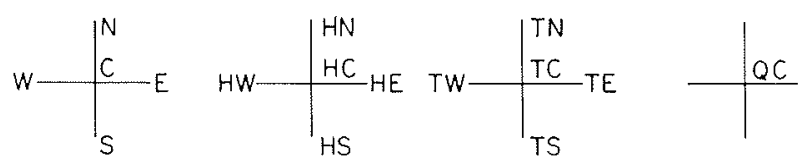

Suivant ce schéma, on écrit pour chacune des $i$ mailles :

$$
\begin{aligned}
\mathrm{Q}_{i} & =\frac{\left(\mathrm{T}_{n}+\mathrm{T}_{c}\right)_{i}}{2}\left(\mathrm{H}_{n}-\mathrm{H}_{c}\right)_{i}+\frac{\left(\mathrm{T}_{s}+\mathrm{T}_{c}\right)_{i}}{2}\left(\mathrm{H}_{s}-\mathrm{H}_{c}\right)_{i} \\
& +\frac{\left(\mathrm{T}_{e}+\mathrm{T}_{c}\right)_{i}}{2}\left(\mathrm{H}_{e}-\mathrm{H}_{c}\right)_{i}+\frac{\left(\mathrm{T}_{w}+\mathrm{T}_{c}\right)_{i}}{2}\left(\mathrm{H}_{w}-\mathrm{H}_{c}\right)_{i}
\end{aligned}
$$

L'inconnue est $\overline{\mathrm{T}}$ qui a donc $(i+f)$ coordonnées. Il y a donc plus d'inconnues que d'équations.

Nous recherchons la solution la plus régulière ne comportant notamment que des composantes positives.

Nous effectuons done une projection de $T$ sur des vecteurs de plus en plus oscillants.

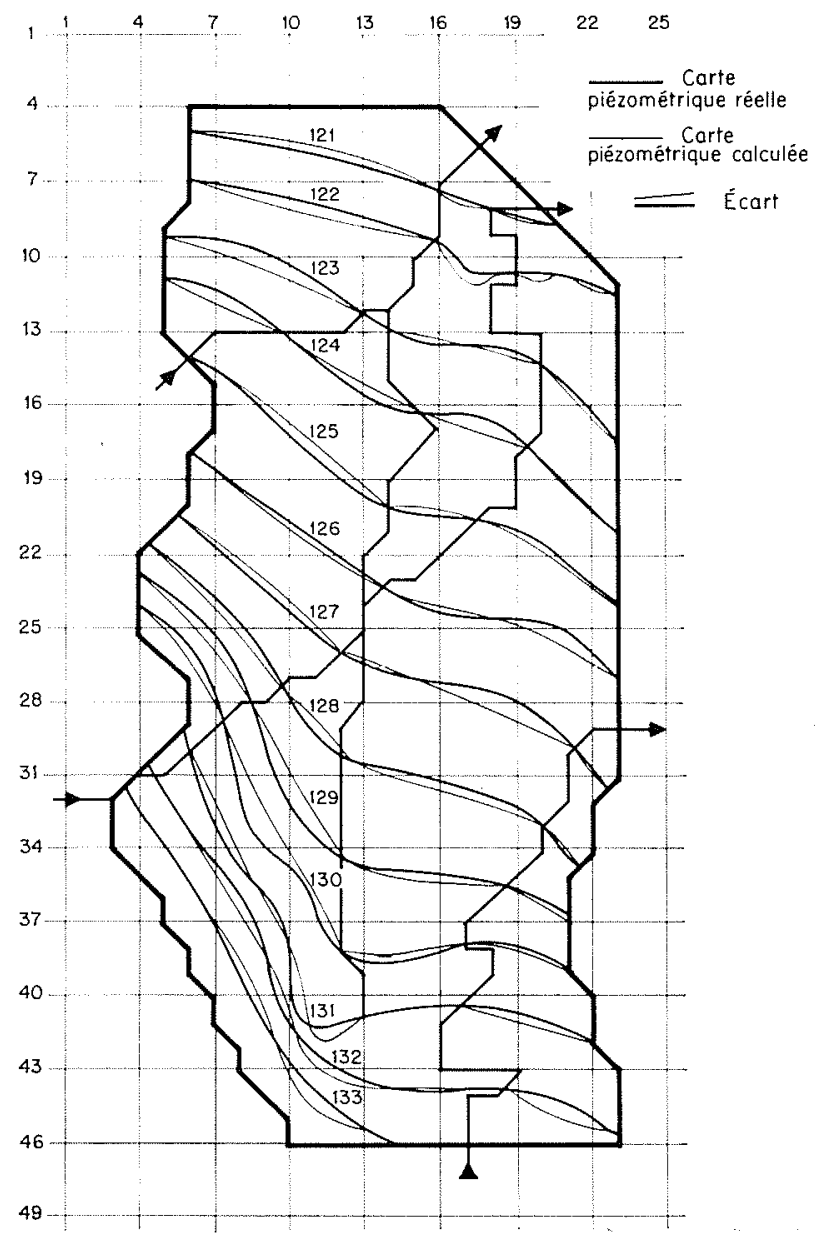

4/ Rhin. Nappe alluviale. Secteur Strasbourg-nord (Transmissivités du "Problème inverse ").

Alluvial aquifer of the Rhine north of Strasbourg ("Reverse problem" transmissibilities).

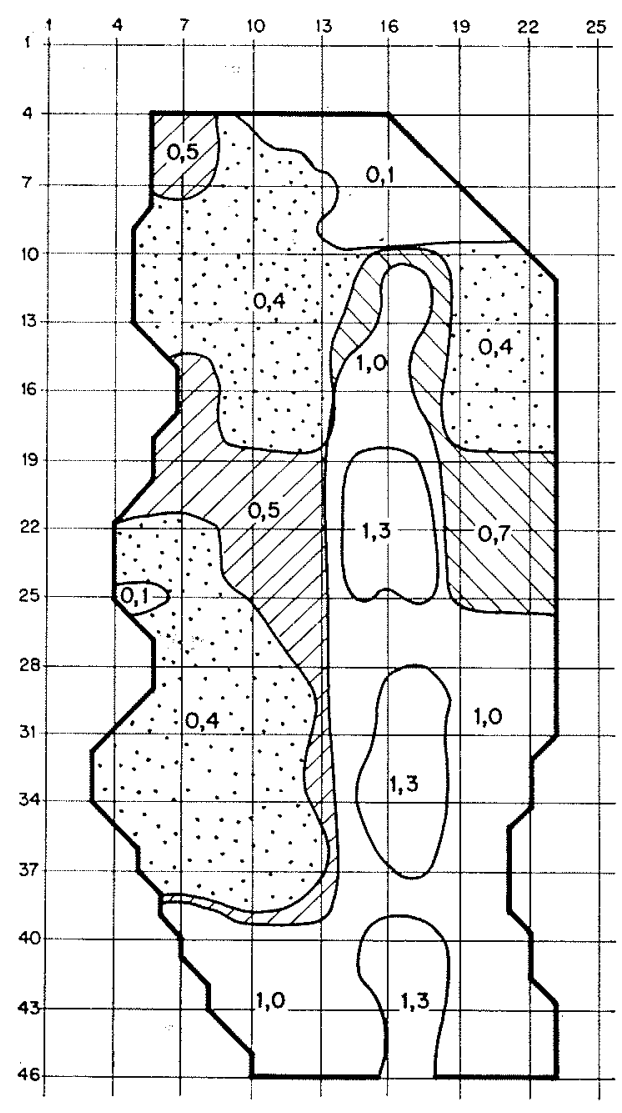

5/ Rhin. Nappe alluviale. Secteur Strasbourg-nord. Transmissivités déterminées par le "Problème inverse ". T en $10^{-1} \mathrm{~m}^{2} / \mathrm{s}$.

Alluvial aquifer north of Strasbourg. Transmissibilities determined by "Reverse problem". $T$ in $10^{-1} \mathrm{~m}^{2} / \mathrm{sec}$. 


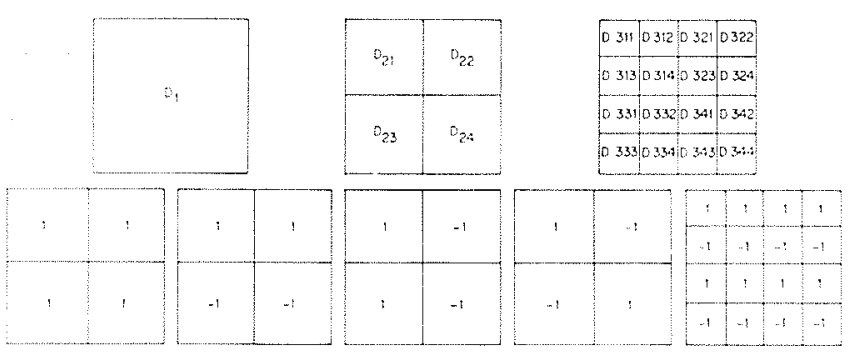

Pour cela nous définissons $\mathrm{E}_{1}$ égal à 1 sur les $i+f$ coordonnées. Partageons le domaine en 4 sous domaines égaux $\mathrm{D}_{21}, \mathrm{D}_{22}, \mathrm{D}_{23}, \mathrm{D}_{24}: \mathrm{E}_{2}, \mathrm{E}_{3}$, $\mathbf{E}_{4}$ ont les valeurs suivantes:

\begin{tabular}{|c|c|c|c|}
\hline & $\mathrm{E}_{2}$ & $\mathrm{E}_{\mathrm{i}}$ & $\mathrm{E}_{4}$ \\
\hline $\mathrm{D}_{21}$ & 1 & 1 & 1 \\
\hline $\mathrm{D}_{22}$ & 1 & -1 & -1 \\
\hline $\mathrm{D}_{30}$ & -1 & -1 & 1 \\
\hline $\mathrm{D}_{2.4}$ & -1 & 1 & -1 \\
\hline
\end{tabular}

Nous poursuivons donc ce découpage de plus en plus fin pour obtenir une base complète. Le problème essentiel réside dans le noyau de déconvolution : $H$ étant donné, il existe en effet $f$ vecteur $\bar{S}_{i}$ tel que :

$$
\text { (S) } \overline{\mathrm{H}}=0
$$

donc ( $\left.\mathrm{T}+a_{1} \mathrm{~S}_{1}+\ldots+a_{f} \mathrm{~S}_{f}\right)$ est solution si (T) est solution.

Or, les $\mathrm{S}_{i}$ ont des composantes fluctuantes, de signes contraires et n'apportent de ce fait aucune information pour notre problème : il s'agit donc de minimiser leur contribution dans la solution, ce que réalise la projection qui n'autorise les composantes fluctuantes à n'intervenir que lorsque le maximum a été fait pour fournir une explication avec des composantes régulières.

\section{Application.}

La méthode a été essayée sur un cas réel. Elle a fourni une bonne concordance entre la simulation de la piézométrie et la réalité, avec un très petit nombre d'itérations.

\section{Le problème direct}

Nous avons de même appliqué la méthode à la résolution du problème direct où $H$ est inconnue :

$$
(\mathrm{T}) * \overline{\mathrm{H}}=\overline{\mathrm{Q}}
$$

Le problème possède alors une solution unique puisque $\bar{H}$ a autant de coordonnées que $\overline{\mathrm{Q}}$. La phase actuelle de travail du laboratoire consiste à comparer les performances de la méthode à celles des méthodes classiques des différences finies ou des éléments finis.

\section{Développement}

Plusieurs orientations sont actuellement retenues.

a) Le problème inverse en régime transitoire.

L'équation s'écrit :

$$
(\mathrm{T}) * \mathrm{H}(t)=(\mathrm{S}) \frac{d \mathrm{H}(t)}{d t}+\mathrm{Q}
$$

$H$ et $Q$ étant connus, ( $\mathrm{T}$ ) et (S) sont calculés en projetant $\overline{\mathrm{T}}$ et $\overline{\mathrm{S}}$, respectivement, sur la même base que précédemment.

\section{b) La recherche des débits inconnus.}

On connaît généralement les débits $Q_{p}$ des puits et des rivières, mais ni l'infiltration ni l'évaporation. Or, $Q_{p}$ et $\overline{\mathrm{H}}$ étant donnés, $Q_{p}$ étant le débit des puits :

$$
(\mathrm{T}) * \mathrm{H}=\mathrm{Q}
$$

ne peut être satisfaisant en général que si :

$$
\mathrm{Q}=\overline{\mathrm{Q}}_{p}+\overline{\mathrm{Q}}_{i}+\overline{\mathrm{Q}}_{e}
$$

$\bar{Q}_{i}=$ infiltration;

$\overline{\mathrm{Q}}_{e}=$ évaporation.

En faisant l'hypothèse que (T) et $\bar{Q}_{e}+Q_{i}$ sont réguliers, on peut donc appliquer à nouveau la méthode en considérant le vecteur généralisé :

$$
\overline{\mathrm{Q}}_{e}+\overline{\mathrm{Q}}_{i}
$$

Les premiers résultats obtenus sux des cas théoriques confirment la possibilité d'un tel ajustement.

c) Recherche simultanée des entrées et de l'opérateur.

$\mathrm{S}(t)$ étant une sortie mesurée, $\mathrm{S} t)=\rho * s(t)$ ne peut ètre satisfait pour $\rho$ et $s$ quelconques, puisque $\rho$ et $s$ sont liées. Si l'on peut admettre que $\rho$ et $s$ sont réguliers, leur ajustement est envisageable à l'aide de la méthode si l'on dispose d'un grand nombre de mesures sur $\mathrm{S}$.

\section{d) Généralisation.}

Pour simplifier l'exposé, nous n'avons envisagé pour les opérateurs à ajuster qu'un nombre de composantes égal à une puissance de 2 : il ne s'agit nullement d'une nécessité. Par exemple, pour 3 composantes, la base est constituée par :

$$
\begin{array}{rrr}
1 & 1 & 1 \\
1 & 0 & -1 \\
1 & -1 & 1
\end{array}
$$

Conclusion

La méthode présentée possède une grande généralité et a fourni des résultats physiquement acceptables pour des problèmes réels non résolus précédemment de facon satisfaisante.

Le Centre d'Informatique Géologique s'attache à présent à développer ses applications. 


\section{Discussion}

Président : M. A. HoupeurT

M. le President remercie et félicite M. EMsErLem et ouvre la discussion.

M. Vachaud roudrait savoir si la methode exposée permet d'espérel la solution du probjeme général de l'évolution quantitative d'une nappe à la suite d'une chute de pluie donne.

Tout dépend, répond M. Exsellem, de la grandeur de maille adoptée et de la valeur des hypothèses faites sur la structure de l'aquifère. On doit pouvoir obtenir au moins une esquisse de ce qui se passe...

En s'aidant de projactions illustrant les résultats obtenus, M. UNGearach décrit, à titre d'exemple, l'étude de la nappe du. Rhin. "Tout d'abord, dit-il, nous avons envisagé des méthodes de simulation directe sur modèle mathématique en regime permanent, ce qui nous a permis d'améliorer la description des transmissivités.

Nous avons également utilisè la méthode exposće par M. Emsellem. Enfin, nous avons eu la curiosité de vouloir restituer une carte de transmissivité à partir des sondages électriques, et, dans le souci de faire sortir la prospection électrique de son cadre habituel, nous avons essayé de voir dans quelle mesure cette méthode pouvait être utilisée pour la determination des paramètres aquifères d'un milieu poreux. Nous avons effectué quelques tests de cohérence sur un modèle mathématique direct, qui ont confirmé le bien fondé de la distribution des transmissivités déduites des résistances transversales corrigées. En particulier, la convergence entre dẻbits d'échanges nappe-rivière calculés et mesurés est particulièrement bonne.

On pourra consulter pour plus de détails les publications suivantes:

1. C. Jain, A. Lagande, P. Chavmet et Y. Emsellem, Interprétation des essais de pompage dans les aquifèles multistrates. Chronique d'hydrogéologie, $\mathrm{N}^{\circ} 12$ (Editions B.R.G.M.).

2. Y. Exsellem et P. Prudhome. - Traitement des mesudes hydrogéologiques à l'aide de modèles. Annales des Mines, $\mathrm{N}^{\circ}$ janvier 1967.

3. Y. Enstrim. - Les transferts de pression entre nappes et la drainance dans les ensembles nquifères hétérogè- nes. Chronique d'Hydrogéologie, No 11, 1967 (Editions B.R.G.M.).

4. Y. Emsellem et G. de Mansur. - Présentation d'une méthode nouvelle de résolution du «problème inverse ». Ecole Nationale Supérieure des Mines de Paris, Centre d'Informatique géologique, nai 1969.

5. P. Ungemach, F. Mostaghimi, A. Duprat. - Essais de détermination du coefficient d'emmagasinement en nappe libre. Application à la nappe alluviale du Rhin. Bull. A.I.H.S., XIV* année, $\mathrm{N}^{\circ} 2$, juin 1969, p. 169-189.

6. Duprat, L. Simler, P. Unglemach. - Contribution de la prospection électrique à la rechorche des caractéristiques hydrodynamiques d'un milieu aquifere (a parấtre dans Annales des Mines).

M. le Président sonligne que tout ce qui concourt à nous renseigner par des moyens directs sur la distribution des transmissivités est du plus haut intérêt. Il demande si la méthode, telle qu'elle a été appliquée, est absolument générale; s'appliquerait-elle à un «feuillage » comportant des passées argileuses sćparant plus ou moins des plans sableux ?

Après un echange de rues entre M. Unaemach et M. DupRAT d'où il ressort que des passées argileuses intermédiaires peuvent être détectées par voie géophysique dans la mesure où les contrastes de l'ésistivité et d'épaisseur vis-àvis des terrains encaissants le permettent (Cf. Theorie du sondage électrique in Kunetz Electrical Soundings Geopublication Associates, 1966), M. LaKshmaNas apporte un debut de confirmation expérimentale; depuis cinq à six ans, ditil, on a tracé des cartes de transmissivité à partir de sondages électriques dans les plaines alluviales de la Saône et de la Garonne. Elles sont utilisées de facon pratique. Done, la méthode empirique, même dans le cas de deux conches alluviales separes par une couche argileuse (il ne s'agit pas d'un feuilletage fin, mais tout de même de deux nappes séparées) donne des résultats positifs.

M. Ie Président clôt la séance en remerciant les conférenciers et les personnes qui, en iniervenant dans la discussion, ont permis un dialogue particulièrement vivant.

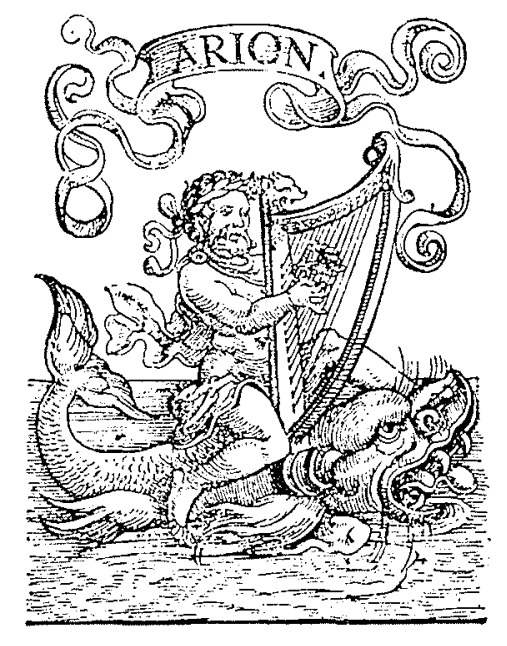




\section{Abstract \\ Automatic restoration of the permeability of an aquifer The inverse problem and deconvolution}

The data interpretation problem frequently arises in the following terms:

A physical system of unknown characteristics has the effect of transforming an input phenonmenon $s$ into an output quantity $\mathrm{S}$.

For example, where constant rates of flow are pumped from a number of wells, the piezometric level of the tapped aquifer is $H(x, y, z)$ when equilibrium is attained.

Similarly, the initial concentration of a tracer penetrating into a porous tube is $c$ ( $t$ ) and, due to diffusion (for example), the concentration of the liquid emerging from the tube is $\mathrm{C}(\mathrm{t})$.

The following questions then arise:

In the first case what must the transmissibility distribution $\mathrm{T}$ be to satisfy

$$
\operatorname{div} \mathrm{T} \operatorname{grad} \mathrm{H}=\mathrm{Q}
$$

And in the second case, what "operator" $p(t)$ expresses the response to an instantaneous injection of a unit mass of tracer $\mathrm{U}$, so that

$$
\mathrm{C}(\mathrm{t})=\int_{0}^{t} p(x) c(t-x) d x
$$

Both problems have the same structure in that data on the operator are required, i.e. on the physical system, which is represented either by a description of what it consists of $(T)$ or by its response to an elementary demand $(p)$.

The method described starts out from the observation that current iterative calculation methods (especially Fourier series) tend to be unstable, typically due to the physically absurd fact that the local mean of the values of several points is very much smaller than the local variation amplitude.

The proposed new method involves an iteration procedure in which the values of the unknown quantity are modified in decreasing steps.

In addition, any punctual modification can only result from an overall modification to the whole of the supporting domain for the unknown quantity.

The proposed method has been applied to deconvolution, automatic restoration of permeability (the reverse problem) and the solution of elliptical equations of the type encountered in hydrodynamics (direct problem).

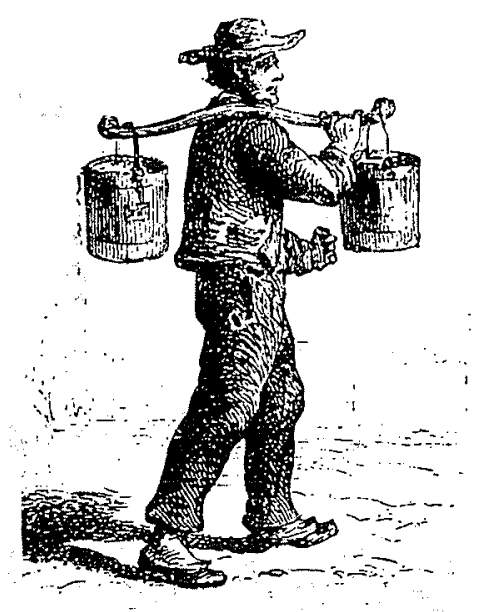

